\title{
Single Event Gate Rupture in 130-nm CMOS Transistor Arrays Subjected to X-Ray Irradiation
}

\author{
Marco Silvestri, Student Member, IEEE, Simone Gerardin, Member, IEEE, Federico Faccio, Member, IEEE, and \\ Alessandro Paccagnella, Senior Member, IEEE
}

\begin{abstract}
We present new experimental results on heavy ion-induced gate rupture on deep submicron CMOS transistor arrays. Through the use of dedicated test structures, composed by a large number of 130-nm MOSFETs connected in parallel, we show the response to heavy ion irradiation under high stress voltages of devices previously irradiated with $\mathrm{X}$-rays. We found only a slight impact on gate rupture critical voltage at a LET of $32 \mathrm{MeV} \mathrm{cm}^{2} \mathrm{mg}^{-1}$ for devices previously irradiated up to $3 \operatorname{Mrad}\left(\mathrm{SiO}_{2}\right)$, and practically no change for $100 \mathrm{Mrad}\left(\mathrm{SiO}_{2}\right)$ irradiation, dose of interest for the future super large hadron collider (SLHC).
\end{abstract}

Index Terms-Gate rupture, heavy ions, SEGR, SLHC, TID, total ionizing dose, ultra-thin gate oxides, X-ray.

\section{INTRODUCTION}

$\mathbf{S}$ INGLE EVENT EFFECTS (SEE) induced by heavy ions present a continual reliability challenge to the operation of the electronic devices used in radiation harsh environments, such as space and high energy physics (HEP) experiments.

The effects produced by heavy ions hitting a sensitive part of an integrated circuit can be complicated, and depend on several factors related to the ion (LET, energy, incident angle) and to the characteristics of sensitive devices. Non-destructive phenomena have been observed such as a bit-flips in an SRAM cell, charge loss from flash memory floating gate, stuck bits, microdose effects, single event functional interrupt (SEFI) [1]. However, heavy ions can induce also destructive events such as burn-out of a power MOSFET, and gate rupture [2]-[4]. The probability of catastrophic events, such as single event gate rupture (SEGR) must be carefully assessed, especially when maintenance is unfeasible, like in spacecrafts and satellites, or quite problematic, like in HEP experiments. In the latter case, thousands to millions of electronics channels operate in tracker detectors very close to the collision point, and reliability must be ensured for many years under extremely high levels of radiation [5].

Manuscript received September 10, 2009; revised November 05, 2009; accepted December 07, 2009. Date of current version August 18, 2010.

M. Silvestri was with the Dipartimento di Ingegneria dell'Informazione, Università di Padova, 35131 Padova, Italy. He is now with Thales Alenia Space Italy, Strada Antica di Collegno 253, 10146 Torino, Italy, and also with the Istituto Nazionale di Fisica Nucleare (INFN), 35131 Padova, Italy (e-mail: marco. silvestri@external.thalesaleniaspace.com).

S. Gerardin and A. Paccagnella are with the Dipartimento di Ingegneria dell'Informazione, Università di Padova, 35131 Padova, Italy, and also with the Istituto Nazionale di Fisica Nucleare (INFN), 35131 Padova, Italy.

F. Faccio is with the Physics Department, Microelectronics Group, CERN, 1211 Geneva 23, Switzerland.

Digital Object Identifier 10.1109/TNS.2009.2039002
The use of modern CMOS devices even below the 130-nm technological node in radiation-harsh environments rises concerns about the long-term reliability due to the continuous increase of the operative electric fields [6]. Concerning total ionizing dose (TID) effects, even if the lateral isolation (STI) still represent the Achilles' heel of modern CMOS devices [7], the very thin gate oxides used nowadays are much less sensitive. Considering SEGR, the sensitivity of modern scaled devices is reduced as well (in spite of the higher electric field) as compared to thicker and older oxides [8] even when new high- $\kappa$ dielectrics are used [9].

Besides the large amount of TID accumulated in the front-end electronics of future HEP experiments, such as the super large hadron collider (SLHC) (up to $100 \mathrm{Mrad}$ in ten years [5]), SEE are expected due to the large number of fast hadrons produced by particle collisions at the center of the experiments. Secondary ions generated by nuclear reactions of fast hadrons with chip and transistors materials [10], may pose a serious threat to the gate oxide of the front-end electronics.

Past works demonstrated that irradiation with $\gamma$-rays below $1 \mathrm{Mrad}\left(\mathrm{SiO}_{2}\right)$ do not impact SEGR occurrence in MOS capacitors [11] and power MOSFETs [12]. Similarly, precursor damage generated by heavy ions was found to have minor influence on SEGR [13], but was shown to contribute to the lifetime reduction of the devices [14], [15].

In contrast, proton irradiation was found to reduce the SEGR critical voltage of power MOSFETs subsequently subjected to heavy ion irradiation [16]. However, tests have never been performed with the high TID levels expected in the SLHC in deep submicron devices, as carried out in this paper.

In this work we present a study of the influence of precursor damage, produced by $10-\mathrm{KeV} \mathrm{X}$ rays up to very high total dose levels, on single event gate rupture in 130-nm CMOS transistors, through the use of dedicated test structures. In particular, we evaluate how the combination of heavy ion irradiation, TID, and high bias impact the integrity of the gate oxide. We will show that the critical voltage to breakdown lies far above the nominal operative bias, and assess if previous X-ray exposure to different levels of TID can change the SEGR critical voltage due to the different distribution and concentration of the generated defects.

\section{EXPERIMENT AND DEVICES}

We performed our experiments with a dedicated test chip defined and designed to asses SEGR on realistic CMOS transistors. Three transistor arrays have been integrated in the chip, each made up of MOSFETs with gate oxide thickness of $2.2 \mathrm{~nm}$, whose source, drain, and bulk were short-circuited, while all the gates were connected in parallel by metal interconnects. Each 
array is designed to have one bonding pad for source, drain, and gate. Close to the gate bonding pad, a double diode protects the structure from plasma damage and electrostatic discharge events.

The three arrays were designed as follows.

1) NMOS-A: NMOSFET array composed of large elements (limits are imposed by design rules in the used technology). The individual element size has been chosen to be $15 \times$ $15 \mu \mathrm{m}^{2} .354$ such elements are connected in parallel, for a total gate area of $79650 \mu \mathrm{m}^{2}$. Each individual gate is connected to a protection diode ( $\mathrm{n}+$ in $\mathrm{p}$-substrate) to prevent plasma damage during manufacturing.

2) NMOS-B: This capacitor has the same total gate area of NMOS-A, but has been designed with a larger number of elements (318600), each with a much smaller gate area of $0.5 \times 0.5 \mu \mathrm{m}^{2}$. These basic elements are grouped in building blocks of 900 , so that the gate area of each is the same as the individual element in NMOS-A $\left(225 \mu \mathrm{m}^{2}\right)$. Each such block has ten protection diodes $(\mathrm{n}+\mathrm{in} \mathrm{p}$-substrate).

3) PMOS-A: This capacitor is geometrically identical to NMOS-A, but in this case the basic transistor is a p-MOS instead of an n-MOS. All protection diodes are the same.

The LHC and SLHC inner tracker radiation environment is mainly characterized by the presence of protons. According to [17], charge yield of $10-\mathrm{keV} \mathrm{X}$ rays more closely matches that of protons than ${ }^{60} \mathrm{Co}$ gamma rays. Moreover, the need to accumulate very high doses in a reasonable amount of time led us to choose the CERN 10-keV X-ray facility. We irradiated the samples with a fixed dose rate of $25 \mathrm{krad}\left(\mathrm{SiO}_{2}\right) / \mathrm{min}$, keeping the devices in the worst-case bias condition during irradiation (all terminals grounded except the gate of the nMOS transistors, kept at $V_{d d}$ ). We measured the $I_{g}-V_{g s}$ before and after the exposure. While some samples were exposed to 30-100 $\mathrm{Mrad}\left(\mathrm{SiO}_{2}\right)$, doses of interest for SLHC applications, others were irradiated to $3 \mathrm{Mrad}\left(\mathrm{SiO}_{2}\right)$, a level of TID known to produce the worst-case response for lateral source-drain leakage current [18].

Both X-ray pre-irradiated and unirradiated samples were then subjected to heavy ion irradiation at the Cyclone Heavy Ion Facility (HIF), Louvain-la-Neuve, Belgium, with 756-MeV Krypton ions (LET $=32.4 \mathrm{MeV} \mathrm{cm} \mathrm{mg}^{-1}$ ) at a fixed flux of $2 \times 10^{4} \mathrm{~s}^{-1} \mathrm{~cm}^{-2}$. During heavy ions experiments the staircase voltage shown in Fig. 1 (with sampling at low gate voltage) was applied at the gate contact [14], [19], [20], keeping the other terminals grounded in order to maintain the samples in inversion. The gate voltage was raised from $\pm 1.5 \mathrm{~V}$ with steps of $100 \mathrm{mV}$, each lasting $25 \mathrm{~s}$ (voltage ramp rate $4 \mathrm{mV} \mathrm{s}^{-1}$ ), until gate-oxide breakdown (BD) occurred. The breakdown voltage $V_{\mathrm{BD}}$ in these tests is taken as the voltage at which $I_{g-\mathrm{LV}}$ suddenly increases (remaining stable) more than one order of magnitude. After the event, a conductive path through the gate dielectric of (at least) one of the transistors in the array is present. During irradiation we monitored the gate current at the stress voltage $\left(V_{g}-\mathrm{HV}\right)$ and periodically (every second) we sampled the gate current at low voltage $\left(V_{g-\mathrm{LV}}= \pm 250 \mathrm{mV}\right)$ in order to detect soft breakdown events [21]. The $V_{g-L V}$ sense voltage does not stress at all the gate oxide, but it is useful to

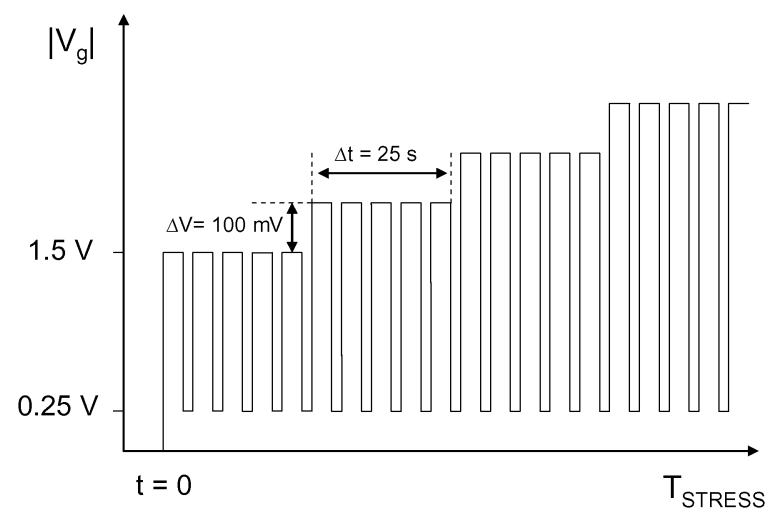

Fig. 1. Simplified representation of the staircase voltage used in our experiments (not to scale). It starts from $1.5 \mathrm{~V}$ and is updated by $100 \mathrm{mV}$ every $25 \mathrm{~s}$ until breakdown occurs. Periodically (every second) we sample the current at $250 \mathrm{mV}$ in order to detect soft breakdown events.

control the low leakage current otherwise masked by the high tunneling current measured at stress voltage $\left(V_{g-\mathrm{HV}}\right)$.

Moreover, before and during heavy ion experiments we stopped the irradiation with a very fast shutter in order to measure the $I_{g}-V_{g s}$ characteristics of the arrays. All measurements were performed with a semiconductor parameter analyzer HP4156B.

\section{X-RAY IRRADIATION RESULTS}

Fig. 2 shows the gate current as a function of the gate voltage $\left(I_{g}-V_{g s}\right)$ for unirradiated and for X-ray irradiated NMOS-A and NMOS-B samples at different total ionizing doses. We show the curve mainly in inversion, because we cannot apply a large negative voltage $\left(\mathrm{V}_{\mathrm{gs}}<\sim-0.5 \mathrm{~V}\right)$ to the gate, in order not to turn on the protection diode.

NMOS-A arrays, presented in Fig. 2(a), exhibit only a slight increment in the leakage current with dose up to $100 \mathrm{Mrad}\left(\mathrm{SiO}_{2}\right)$, visible at very low gate voltages (100-200 mV). As for NMOS-B [Fig. 2(b)] the current of the irradiated samples overlaps the one of the unirradiated ones for higher gate voltages (above $0.4 \mathrm{~V}$ ).

In contrast NMOS-B arrays presented in Fig. 2(b) display a large increment in the gate leakage current of about three orders of magnitude at $250 \mathrm{mV}$ after $30 \mathrm{Mrad}\left(\mathrm{SiO}_{2}\right)$. The leakage current measured after $100 \mathrm{Mrad}\left(\mathrm{SiO}_{2}\right)$ is almost one order of magnitude below the one taken after $30 \mathrm{Mrad}\left(\mathrm{SiO}_{2}\right)$, and close to that after $3 \mathrm{Mrad}\left(\mathrm{SiO}_{2}\right)$. Moreover at low voltage the leakage current changes sign, and the voltage at which this occurs moves toward more positive gate voltage values (about $15 \mathrm{mV}$ ) after irradiation. A larger shift of that voltage (current inversion) is observed in the $I_{g}-V_{g s}$ of PMOS-A devices as a function of TID, as shown in Fig. 3. We found about $250 \mathrm{mV}$ and $500 \mathrm{mV}$ after 3 and $100 \mathrm{Mrad}\left(\mathrm{SiO}_{2}\right)$, respectively. Again the current exhibited by irradiated samples rejoins the one related to unirradiated ones at higher gate voltages.

We want to remark that for large enough gate voltages, no radiation induced leakage current is visible. This behavior is representative of at least three samples for each dose level and array structure. 

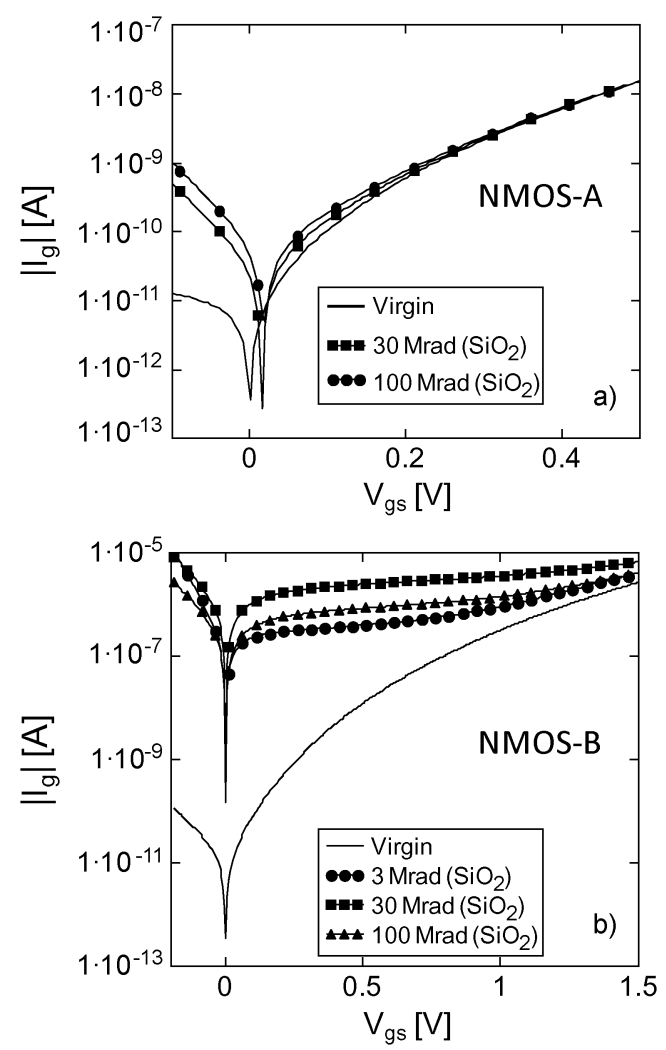

Fig. 2. Gate leakage current as a function of the gate voltage for different amount of X-ray exposure in (a) NMOS-A and (b) NMOS-B samples.

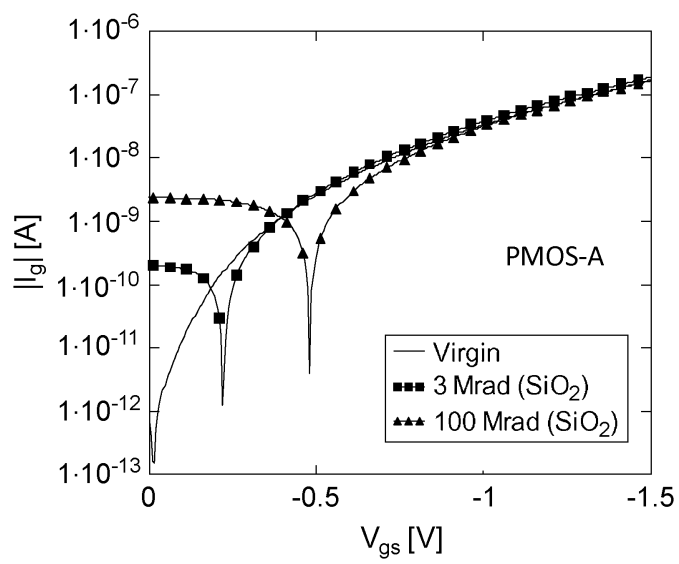

Fig. 3. Gate leakage current as a function of the gate voltage for different amount of X-ray exposure in PMOS-A samples.

\section{HEAVy IONS IRRADIATION RESUlTS}

\section{A. Samples Not Irradiated With X-Rays}

Fig. 4(a) shows the gate leakage current taken at low sense voltage $\left(I_{g-\mathrm{LV}}\right.$ at $\left.250 \mathrm{mV}\right)$ as a function of stress time for an NMOS-A device measured outside of the beam and one under heavy ions beam. Correspondingly in Fig. 4(b) the $I_{g-\mathrm{LV}}$ is reported for NMOS-B devices. In both plots the transistor arrays were not previously irradiated with X-rays.

The breakdown event occurs earlier under heavy ion irradiation, displaying $100-\mathrm{mV}$ reduction in the rupture voltage $\left(V_{\mathrm{BD}}\right)$ for both structures. Neither progressive breakdown (PBD) nor
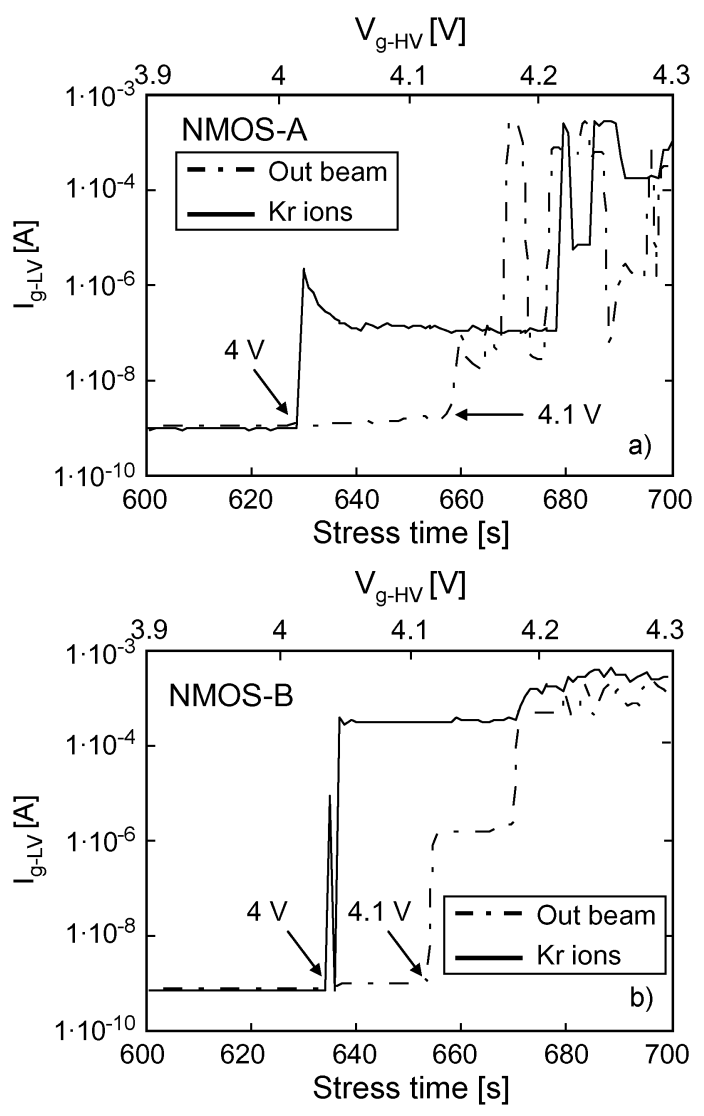

Fig. 4. Gate leakage current (taken at $250 \mathrm{mV}$ ) as a function of stress time and gate voltage for devices stressed outside and under $\mathrm{Kr}$ ion beam not previously irradiated with X-rays. (a) NMOS-A samples and (b) NMOS-B samples.

leakage current increase were detected, and both NMOS-A and NMOS-B exhibited a soft breakdown $\left(\sim 10^{-6} \mathrm{~A}\right)$ followed by a hard one $\left(\sim 10^{-3} \mathrm{~A}\right)$.

On the contrary, there is a slight increase $(<10 \%)$ in the gate current in the devices stressed outside of the beam that starts when the gate ramp has reached $4 \mathrm{~V}$.

Unlike the nMOS devices, pMOS samples (not reported) exhibit a leakage current increase before breakdown during stress both outside and inside the heavy ion beam without any relevant effect. Moreover, heavy ions do not influence the $V_{\mathrm{BD}}$ significantly.

\section{B. X-Ray Irradiated Samples}

Fig. 5 shows the $I_{g-\mathrm{LV}}$ during heavy ion exposure of unirradiated samples reported with dotted lines (solid line in Fig. 4) and previously X-ray irradiated ones up to $100 \mathrm{Mrad}\left(\mathrm{SiO}_{2}\right)$, NMOS-A [Fig. 5(a)] and NMOS-B [Fig. 5(b)] samples. As shown in Fig. 2, and reported in Section III, the effect of total dose on the gate leakage is remarkable only in NMOS-B arrays featuring a leakage current of $10^{-7} \mathrm{~A}$ instead of $10^{-9} \mathrm{~A}$ (taken at $250 \mathrm{mV}$ ) exhibited by fresh devices. On the other hand X-ray irradiated NMOS-A devices [Fig. 5(a)] display only about $1 \times 10^{-9}$ A more leakage current as compared to unirradiated ones. Although the TID effects are visible and not negligible (at low voltage) they do not influence the breakdown occurrence even after $100 \mathrm{Mrad}\left(\mathrm{SiO}_{2}\right)$. In fact we found the same $V_{\mathrm{BD}}(4 \mathrm{~V})$ under heavy ions with and without previous X-ray 


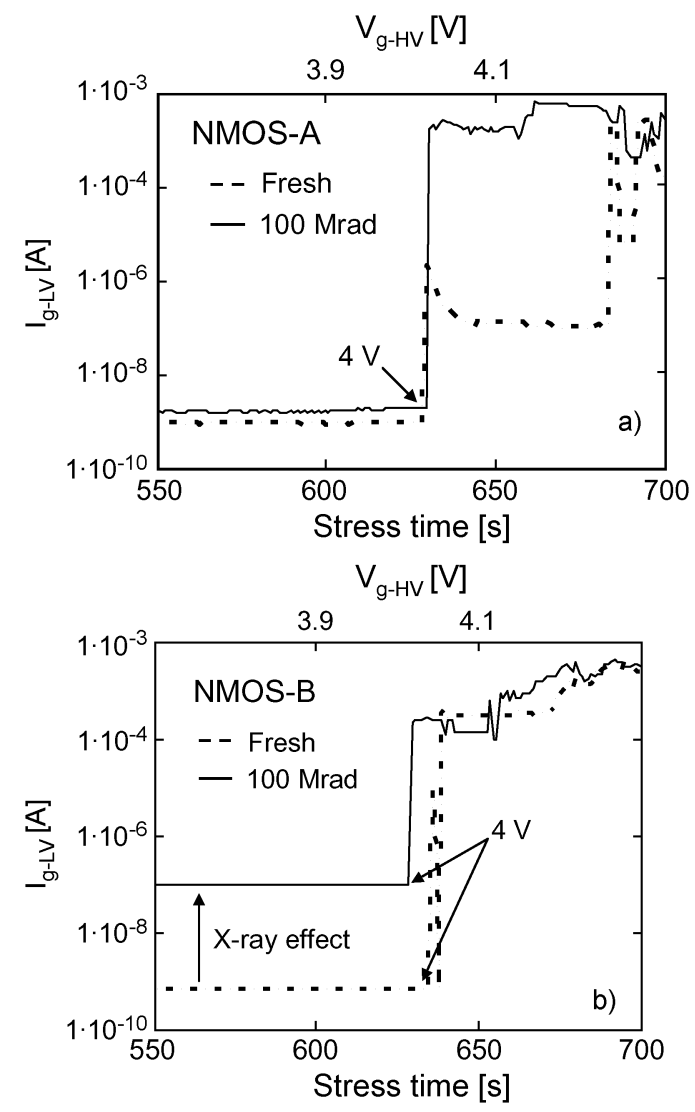

Fig. 5. Gate leakage current (taken at $250 \mathrm{mV}$ ) as a function of stress time and gate voltage for unirradiated and previously irradiated with $\mathrm{X}$-rays up to $100 \mathrm{Mrad}\left(\mathrm{SiO}_{2}\right)$ stressed under heavy ion beam. (a) NMOS-A samples and (b) NMOS-B samples.

irradiation, with a difference in time between the ruptures of few seconds.

Fig. 6 reports the results for NMOS-B samples previously irradiated with $\mathrm{X}$-rays up to $3 \mathrm{Mrad}\left(\mathrm{SiO}_{2}\right)$. As opposed to the $100 \mathrm{Mrad}\left(\mathrm{SiO}_{2}\right)$ X-ray irradiation shown in Fig. 5(b), the $3 \mathrm{Mrad}\left(\mathrm{SiO}_{2}\right)$ exposure leads to an earlier degradation under heavy ions. The hard rupture in X-ray irradiated samples happens between $3.8 \mathrm{~V}$ and $3.9 \mathrm{~V}$, about 100-200 mV before the rupture of the device irradiated with heavy ions but with no previous TID exposure.

The sudden current drop visible with the long dashed line around $620 \mathrm{~s}$ is due to melting of the gate interconnection which is not designed to carry high currents that changes the series resistance and consequently the measured current.

On the contrary, the temporary increase exhibited by the other sample (short dashed line) is a transient conduction through the gate dielectric probably due to unstable defects, often observed before breakdown [22].

X-ray irradiated pMOS arrays do not display appreciable influence on $V_{\mathrm{BD}}$ neither at $3 \mathrm{Mrad}\left(\mathrm{SiO}_{2}\right)$ nor after $100 \mathrm{Mrad}\left(\mathrm{SiO}_{2}\right)$.

We want to point out that the results presented in Sections IV-A and IV-B are representative of all the samples we tested. The slight $V_{\mathrm{BD}}$ differences we found (5\%-7\%) may grow with higher ion LET, according to our previous published data on similar 130-nm CMOS technology [19].

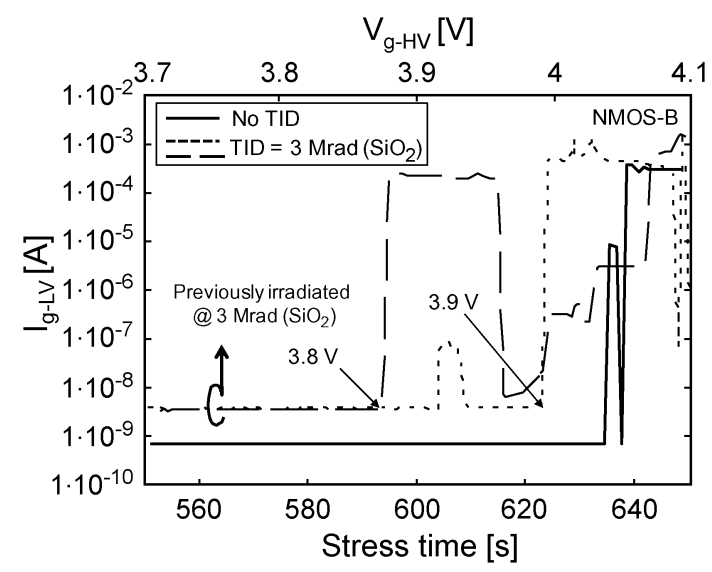

Fig. 6. Gate leakage current (taken at $250 \mathrm{mV}$ ) as a function of stress time and gate voltage for NMOS-B arrays unirradiated and previously irradiated with $\mathrm{X}$-rays up to $3 \mathrm{Mrad}\left(\mathrm{SiO}_{2}\right)$ subsequently stressed under heavy ion beam.

However, even if we are confident about our conclusions, the experimental uncertainty is comparable to the observed differences.

\section{DISCUSSION}

The use of test structures as close as possible to real CMOS transistors was recommended to have a realistic assessment of SEGR issues in submicron CMOS technology [19]. However, the structure we used in that work was still different from real transistors because the polysilicon fingered capacitors were shrunk only in one dimension (the "channel" length was $130 \mathrm{~nm}$ ) while the width was about $100 \mu \mathrm{m}$.

In this work we used transistor arrays designed with a large number of 130-nm CMOS transistors close together with source and drain shorted by design and with the gates connected in parallel. These structures allows us to perform a SEGR test on a large number of theoretically identical devices at the same time, increasing in this way the probability of an ion strike in the gate region.

We have shown in Fig. 4 the comparison between NMOS-A and NMOS-B samples (without previous X-rays) stressed outside and under the heavy ion beam. The small difference between the $V_{\mathrm{BD}}$ obtained with and without $\mathrm{Kr}$ ions may suggests that we are dealing with time-dependent dielectric breakdown phenomenon [23] rather than a single effect induced by a heavy ion. Indeed, the high applied voltage at the gate contact leads to injection of energetic carriers through the gate oxide due to the well known Fowler-Nordheim tunneling mechanisms. The energy released by the interaction between those carriers and the lattice generates defects [23], which could add up to those created by the impinging ions. However, the degradation observed in these experiments is different from previous tests [19], [20], where a clear increase in the leakage current was observed before the hard breakdown due to potential synergism between damage induced by ions and the stress voltage.

In order to understand the influence of the rate at which the gate voltage increases during these SEGR tests we used two different rates: the slow ramp (SR) was $1 \mathrm{mV} \mathrm{s}^{-1}(100 \mathrm{mV}$ every $100 \mathrm{~s})$ and the fast ramp (FR) was $4 \mathrm{mV} \mathrm{s}^{-1}(100 \mathrm{mV}$ every $25 \mathrm{~s}$ ). The number of ions that struck the whole array area 

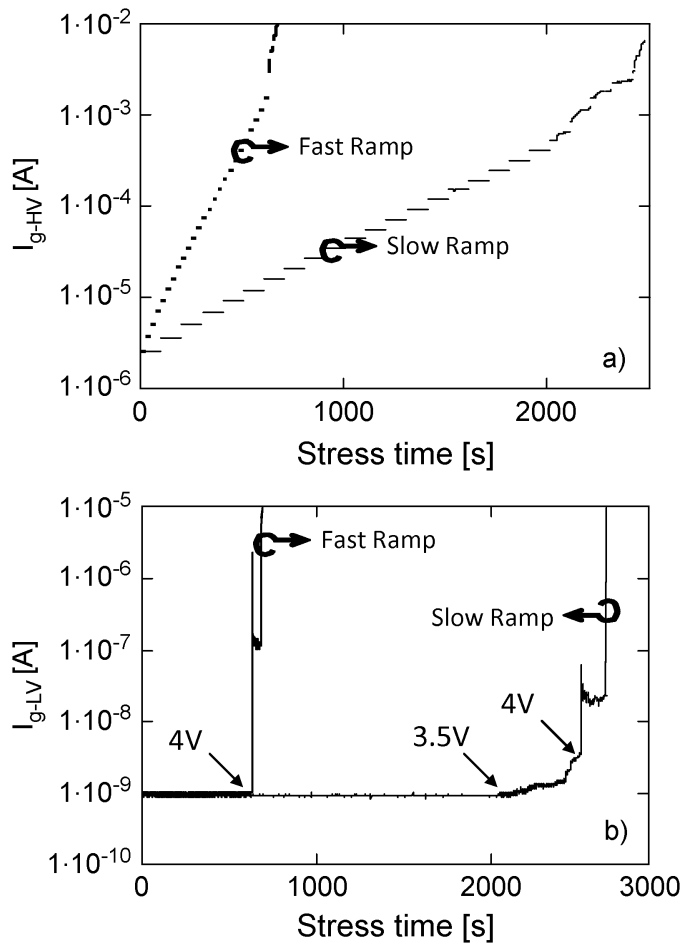

Fig. 7. Gate leakage current as a function of stress time for a NMOS-A stressed with two different gate ramp rates under heavy ions: (a) taken at stress voltage and (b) taken at $250 \mathrm{mV}$ (see Fig. 1).

(including source and drain overlaps of each transistor in the array) during every SR-step (i.e., every $100 \mathrm{~s}$ ) is about 1600 . On average about $4 \times 10^{4} \mathrm{Kr}$ ions hit the array before the onset of the breakdown. The number of ions that strike the entire available gate area (including source and drain overlaps of each transistor in the array) during every FR-step (i.e., every $25 \mathrm{~s}$ ) is about 400; and on average about $9600 \mathrm{Kr}$ ions hit the entire array before breakdown.

Multiple hits in the single MOSFET gate area cannot be excluded, especially for NMOS-A arrays designed with $15 \times 15 \mu \mathrm{m}^{2}$ MOSFETs. Considering a track radius of 100 nm [24], 400 and 1600 strikes per step lead to zero and about one couple of strikes closer than $100 \mathrm{~nm}$, respectively.

As a consequence, overlapping hits can occur mainly when SR-stress rather than FR-stress is used.

The comparison presented in Fig. 7 shows a different behavior when we applied the SR as opposed to the FR during heavy ions exposure. While the breakdown is abrupt with the $\mathrm{FR}$, in the case of the SR the low voltage leakage current $I_{g-\mathrm{LV}}$ as well as the stress current $I_{g-\mathrm{HV}}$ increase progressively before the final rupture, starting from $3.5 \mathrm{~V}(2000 \mathrm{~s})$.

In Fig. 8, we report in detail the comparison between two SR experiments performed with one device under $\mathrm{Kr}$ ions (Fig. 7) and another one out of the beam for reference. No increase in $I_{g-\mathrm{LV}}$ is detected until $2000 \mathrm{~s}(3.5 \mathrm{~V})$ for both devices suggesting that the density of ions-induced and stress-induced defects is not enough to affect the integrity of the gate oxide. However, something happens around $2040 \mathrm{~s}(3.5 \mathrm{~V})$ and the device under heavy ions starts to degrade progressively until the soft breakdown (SBD) and the hard breakdown (HBD) occur around

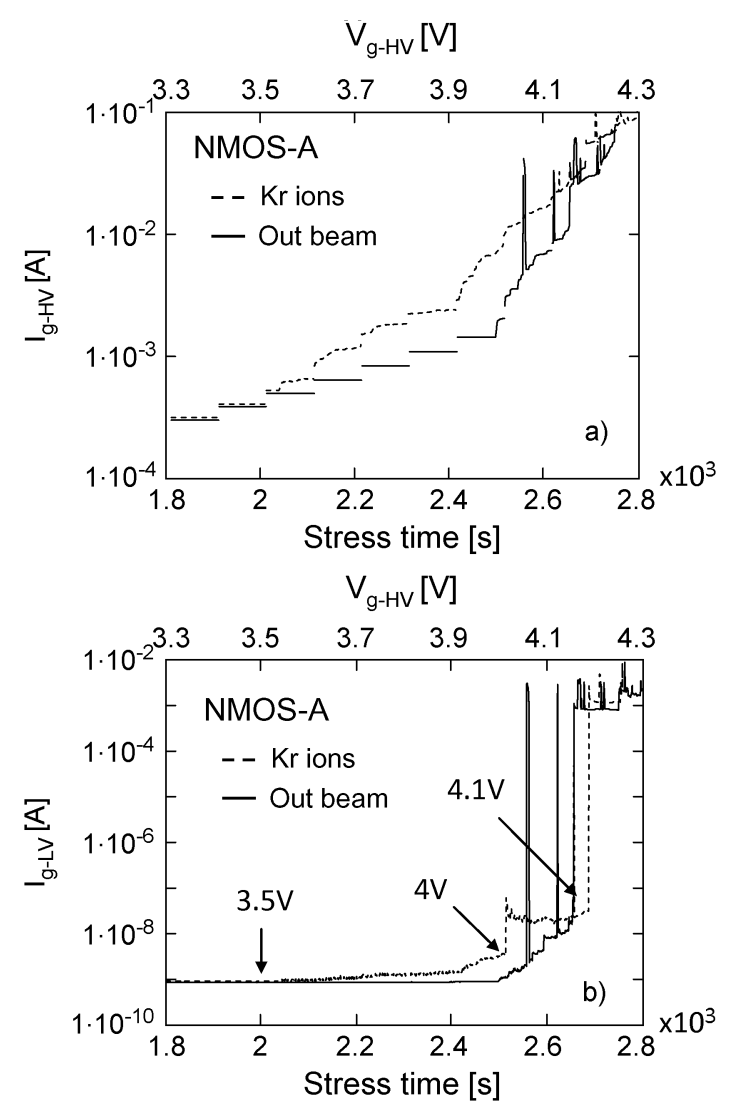

Fig. 8. Gate leakage current as a function of stress time and gate voltage for a NMOS-A stressed outside of the beam and under $\mathrm{Kr}$ ions during slow ramp (SR) experiments: a) taken at stress voltage, b) taken at $250 \mathrm{mV}$ (see Fig. 1).

$2500 \mathrm{~s}(4 \mathrm{~V})$ and $2680 \mathrm{~s}(4.1 \mathrm{~V})$, respectively. In the meantime, the sample stressed outside of the beam reaches about $2500 \mathrm{~s}$ $(4 \mathrm{~V})$ with no gate current increase, then it breaks down progressively accordingly to what has been found in similar ultrathin oxides [25], until $2650 \mathrm{~s}$ (4.1 V) when the intrinsic HBD occurs (a similar behavior is found, to a lesser extent, in the NMOS-B samples where the degradation starts from 3.6 V).

Similarly, the devices tested outside of the beam during FR stresses show the $I_{g-\mathrm{LV}}$ increase (less than $10 \%$ ) only after $4 \mathrm{~V}$ reaching the intrinsic HBD at $4.1 \mathrm{~V}$ (see Fig. 4). However, we want to emphasize that all the FR experiments under heavy ions exhibit a sudden rupture of the gate dielectric with no leakage current progression.

As a consequence, from the electrical stress point of view the comparison between the two procedures suggests that these two different ramp rates do not influence appreciably the SEGR occurrence, since the reference devices (stressed outside of the beam) starts to degrade only after $\sim 4 \mathrm{~V}$ regardless of the $V_{g-\mathrm{HV}}$ rate (compare Figs. 4 and 8).

In contrast, the longer the stress step (slow ramp) the larger the cumulative damage produced by ions as demonstrated in Fig. 8 (higher fluence and double hits per step). These results lead us to assume that the FR-stress limits the influence of cumulative damage produced by the passage of the heavy ions helping us to better discriminate the influence of pre-existing X-ray-induced defect on SEGR incidence. 
As shown in Figs. 2 and 3 we can definitely observe that $\mathrm{X}$-ray irradiation has affected the arrays. In fact, the gate leakage current increases due to the presence of traps in the $\mathrm{SiO}_{2}$ bandgap that favor the carrier tunneling through the gate oxide, deeply investigated and known as radiation induced leakage current (RILC) [26], [27].

However, it is worth highlighting how the amount of leakage current exhibited by NMOS-B samples in Fig. 2(b) is larger than what has been previously published for irradiated capacitors with thicker oxides [26]. Also the rebound in the gate leakage current is in contrast with previous results, where a monotonic degradation with TID was reported. This rebound, though, reflects the behavior of the drain-source leakage current $\left(I_{\mathrm{off}}\right)$ that was found especially in narrow transistors [18]. This inconsistency with previous data can be justified by the difference in the test structures: whilst in [26] large area CMOS capacitors were used (gate area $10^{-2} \mathrm{~cm}^{2}$ ), this work uses arrays of real deep-submicron CMOS transistors, each surrounded by lateral oxide isolation (STI) that after X-ray irradiation, traps charge and develops interfaces states that influence the electrostatics of the transistors. For the same 130-nm technology, the threshold voltage shift induced by X-ray irradiation was found to be maximized around 1-3 $\mathrm{Mrad}\left(\mathrm{SiO}_{2}\right)$ [18].

The comparison between NMOS-A and NMOS-B reported in Fig. 7.2 suggests the presence of a parasitic leakage path through the NMOS-B array. However, all the X-ray irradiated devices exhibit the behavior reported in Fig. 7.2, hence we are dealing with a systematic degradation of the $B$ arrays. As a consequence, the current exhibited after irradiation (at low voltage) is a sum of RILC and parasitic leakage that however has not a significant impact on single event gate rupture, since at the stress voltage $\left(V_{g s}>1.5 \mathrm{~V}\right)$ the gate current is the same regardless of the previous irradiation.

It is interesting to point out that at the very high dose of $100 \mathrm{Mrad}\left(\mathrm{SiO}_{2}\right)$ we do not observe remarkable effects during $\mathrm{Kr}$ ion experiments (see Fig. 5). There is no leakage current increase before breakdown and the $V_{\mathrm{BD}}$ is $4 \mathrm{~V}$ as for the arrays not previously irradiated with X-rays. We only found an earlier breakdown (few seconds) but we cannot attribute it to the X-ray-induced defects. Concerning the $3 \mathrm{Mrad}\left(\mathrm{SiO}_{2}\right)$ irradiated samples, whose results are reported in Fig. 6, we found a more clear earlier breakdown around $3.8 \mathrm{~V}$ and $3.9 \mathrm{~V}$. Even if the breakdown definitely occurred at lower $V_{g s}$, the $5 \%-7 \% V_{\mathrm{BD}}$ difference exhibited at this LET, as compared to devices stressed outside of the beam, may fall within the experimental error. However, 200-300 $\mathrm{mV}$ lower $V_{\mathrm{BD}}$ is in agreement with the results obtained with 212-MeV Nickel ions $\left(\mathrm{LET}=28.2 \mathrm{MeV} \mathrm{cm} \mathrm{mg}^{-1}\right.$ ) in a similar 130-nm technology [19].

Since the gate current measured at stress voltage $\left(V_{g s}>\right.$ $1.5 \mathrm{~V}$ ) is not affected by X-ray irradiation as seen in Figs. 2 and 3 we cannot ascribe the observed $V_{\mathrm{BD}}$ differences to the stress current itself. In fact, a different injection rate of charge during stress can lead to a later or earlier breakdown [28]. In this case, the reason for this earlier BD may be attributed to the different concentration of positive trapped charge and interface states present in the structures before heavy ion exposure, as pointed out in [11], [16]. The presence or absence of a spatial distribution of charge induced by X-ray irradiation in the device can lead to a different local electric field in the oxide, in particular close to the transistors edges. In fact, at this dose rate, a high level of TID in CMOS MOSFETs is mainly characterized by the presence of interface states while positive trapped charge is the main phenomenon at low doses, even if the concentration is low for gate oxide thickness below $3 \mathrm{~nm}$ [7].

The critical defect density to onset the breakdown $d_{\text {crit-BD }}$ is given by the sum of ion-induced defects $d_{\text {heavyions }}$ and applied bias induced ones $d_{\text {bias }}$. When $d_{\text {crit-BD }}$ is reached, a conductive path through the gate oxide is formed, and the current is then able to flow inducing the thermal runaway and finally the breakdown [23]. With previous X-ray exposure, the defect generation rate is possibly modulated by the presence of positive trapped charge and interface states (generated by $\mathrm{X}$ rays) that locally change the net oxide electric field. As a consequence, the electric field enhancement generated by positive charge trapping may cause the breakdown voltage to slightly decrease at 3 $\operatorname{Mrad}\left(\mathrm{SiO}_{2}\right)$. On the contrary, after $100 \mathrm{Mrad}\left(\mathrm{SiO}_{2}\right)$, the larger number of negatively charged interface states compensate the positive trapped charge at the bulk-oxide and bulk-STIs interfaces, possibly reducing the local oxide electric field and consequently the impact on $V_{\mathrm{BD}}$.

\section{CONCLUSION}

In conclusion we found only a slight impact on gate rupture critical voltage at a LET of $32 \mathrm{MeV} \mathrm{cm}^{2} \mathrm{mg}^{-1}$ for devices previously irradiated up to $3 \mathrm{Mrad}\left(\mathrm{SiO}_{2}\right)$, and practically no change for $100 \mathrm{Mrad}\left(\mathrm{SiO}_{2}\right)$ irradiation, which simulates ten years of operation in the future super large hadron collider. We attribute this effect to possible interplay between trapped charge, and defects introduced by X rays, heavy ions, and electrical stress. The rupture voltage lies far from operative conditions even when the devices were subjected to extremely high TID. The results presented in this work, related to the role of border regions, advocate the use of test structures based on MOSFETs arrays, rather than large area capacitors usually used in similar assessments.

\section{REFERENCES}

[1] G. Cellere and A. Paccagnella, "A review of ionizing radiation effects in floating gate memories," IEEE Trans. Device Mater. Rel., vol. 4, no. 3, pp. 359-370, Sep. 2004.

[2] A. H. Johnston, G. M. Swift, T. Miyahira, and L. D. Edmonds, "Breakdown of gate oxides during irradiation with heavy ions," IEEE Trans. Nucl. Sci., vol. 5, no. 6, pp. 2500-2508, Dec. 1998.

[3] M. Allenspach, C. Dachs, G. H. Johnson, R. D. Schrimpf, E. Lorfèvre, J. M. Palau, J. R. Brews, K. F. Galloway, J. L. Titus, and C. F. Wheatley, "SEGR and SEB in n-channel power MOSFETs," IEEE Trans. Nucl. Sci., vol. 43, no. 6, pp. 2927-2931, Dec. 1996.

[4] L. E. Selva, L. Z. Scheick, S. McClure, T. Miyahira, S. M. Guertin, S. K. Shah, L. D. Edmonds, and J. D. Patterson, "Catastrophic SEE in high-voltage power MOSFETs," in IEEE Radiation Effects Data Workshop, 2003.

[5] A. Candelori, "Semiconductor materials and detectors for future very high luminosity colliders," IEEE Trans. Nucl. Sci., vol. 52, no. 6, pp. 2554-2561, Dec. 2005.

[6] R. H. Dennard, J. Cai, and A. Kumar, "A perspective on today's scaling challenges and possible future directions," Solid-State Electron., vol. 51, pp. 518-525, 2007.

[7] H. J. Barnaby, "Total-ionizing-dose effects in modern CMOS technologies," IEEE Trans. Nucl. Sci., vol. 53, no. 6, pp. 3103-3121, Dec. 2006.

[8] F. W. Sexton, "Destructive single-event effects in semiconductor device and ICs," IEEE Trans. Nucl. Sci., vol. 50, no. 3, pp. 603-621, Jun. 2003. 
[9] L. W. Massengill, B. K. Choi, D. M. Fleetwood, R. D. Schrimpf, K. F. Galloway, M. R. Shaneyfelt, T. L. Meisenheimer, P. E. Dodd, J. R. Schwank, Y. M. Lee, R. S. Johnson, and G. Lucovsky, "Heavyion-induced breakdown in ultrathin gate oxides and high-k dielectrics," IEEE Trans. Nucl. Sci., vol. 48, no. 6, pp. 1904-1912, Dec. 2001.

[10] M. J. Beck, B. R. Tuttle, R. D. Schrimpf, D. M. Fleetwood, and S. T. Pantelides, "Atomic displacement effects in single-event gate rupture," IEEE Trans. Nucl. Sci., vol. 55, no. 6, pp. 3025-3031, Dec. 2008.

[11] G. K. Lum, N. Boruta, J. M. Baker, L. Robinette, M. R. Shaneyfelt, J. R. Schwank, P. E. Dodd, and J. A. Felix, "New experimental findings for single-event gate rupture in MOS capacitors and linear devices," IEEE Trans. Nucl. Sci., vol. 51, no. 6, pp. 3263-3269, Dec. 2004.

[12] J. L. Titus and C. F. Wheatley, "Experimental studies of single-event gate rupture and burnout in vertical power MOSFET's," IEEE Trans. Nucl. Sci., vol. 43, no. 2, pp. 533-545, Apr. 1996.

[13] F. W. Sexton, D. M. Fleetwood, M. R. Shaneyfelt, P. E. Dodd, G. L. Hash, L. P. Schanwald, R. A. Loemker, K. S. Krisch, M. L. Green, B. E. Weir, and P. J. Silverman, "Precursor ion damage and angular dependence of single event gate rupture in thin oxides," IEEE Trans. Nucl. Sci., vol. 45, no. 6, pp. 2509-2518, Dec. 1998.

[14] A. Cester, S. Cimino, A. Paccagnella, G. Ghibaudo, G. Ghidini, and J. Wyss, "Accelerated wear-out of ultrathin gate oxides after irradiation," IEEE Trans. Nucl. Sci., vol. 50, no. 3, pp. 729-734, Jun. 2003.

[15] B. K. Choi, D. M. Fleetwood, R. D. Schrimpf, L. W. Massengill, K. F. Galloway, M. R. Shaneyfelt, T. L. Meisenheimer, P. E. Dodd, J. R. Schwank, Y. M. Lee, R. S. Johnson, and G. Lucovsky, "Long-term reliability degradation of ultrathin dielectric films due to heavy-ion irradiation," IEEE Trans. Nucl. Sci., vol. 49, no. 6, pp. 3045-3050, Dec. 2002.

[16] L. Z. Scheick and L. E. Selva, "Effect of dose history on SEGR properties of power MOSFETs," IEEE Trans. Nucl. Sci., vol. 54, no. 6, pp. 2568-2575, Dec. 2007.

[17] P. Paillet, J. R. Schwank, M. R. Shaneyfelt, V. Ferlet-Cavrois, R. L. Jones, O. Flament, and E. W. Blackmore, "Comparison of charge yield in MOS devices for different radiation sources," IEEE Trans. Nucl. Sci., vol. 49, no. 6, pp. 2656-2661, Dec. 2002.

[18] F. Faccio and G. Cervelli, "Radiation-induced edge effects in deep submicron CMOS transistors," IEEE Trans. Nucl. Sci., vol. 52, no. 6, pp. 2413-2420, Dec. 2005.
[19] M. Silvestri, S. Gerardin, A. Paccagnella, and G. Ghidini, "Gate rupture in ultrathin gate oxides irradiated with heavy ions," IEEE Trans. Nucl. Sci., vol. 56, no. 4, pp. 1964-1970, Aug. 2009.

[20] S. Gerardin, A. Cester, A. Paccagnella, G. Gasiot, P. Mazoyer, and P. Roche, "Radiation-induced breakdown in $1.7 \mathrm{~nm}$ oxynitrided gate oxides," IEEE Trans. Nucl. Sci., vol. 52, no. 6, pp. 2210-2216, Dec. 2005.

[21] J. F. Conley, Jr., J. S. Suehle, A. H. Johnston, B. Wang, T. Miyahara, E. M. Vogel, and J. B. Bernstein, "Heavy-ion-induced soft breakdown of thin gate oxides," IEEE Trans. Nucl. Sci., vol. 48, no. 6, pp. 1913-1916, Dec. 2001

[22] R. Degraeve, G. Groeseneken, R. Bellens, J. L. Ogier, M. Depas, P. J. Roussel, and H. E. Maes, "New insights in the relation between electron trap generation and the statistical properties of oxide breakdown," IEEE Trans. Electron Devices, vol. 45, no. 4, pp. 904-911, Apr. 1998.

[23] J. H. Stathis, "Physical and predictive models of ultrathin oxide reliability in CMOS devices and circuits," IEEE Trans. Device Mater. Rel., vol. 1, no. 1, pp. 43-59, Mar. 2001.

[24] P. E. Dodd, "Physics-based simulation of single-event effects," IEEE Trans. Device Mater. Rel., vol. 5, no. 3, pp. 343-357, Sep. 2005.

[25] F. Monsieur, E. Vincent, G. Pananakakis, and G. Ghibaudo, "Wearout, breakdown occurrence and failure detection in $18-25 \AA$ ultrathin oxides," Microelectron. Reliab., vol. 41, pp. 1035-1039, 2001.

[26] M. Ceschia, A. Paccagnella, S. Sandrin, G. Ghidini, J. Wyss, M. Lavale, and O. Flament, "Low field leakage current and soft breakdown in ultrathin gate oxides after heavy ions, electrons or X-ray irradiation," in Proc. IEEE 5th Eur. Conf. Radiation Its Effects on Components Systems, 1999, pp. 233-240.

[27] L. Larcher, A. Paccagnella, M. Ceschia, and G. Ghidini, "A model of radiation induced leakage current (RILC) in ultrathin gate oxides," IEEE Trans. Nucl. Sci., vol. 46, no. 6, pp. 1553-1561, Dec. 1999.

[28] A. Martin, P. O'Sullivan, and A. Mathewson, "Correlation of $\mathrm{SiO}_{2}$ lifetimes from constant and ramped voltage measurements," Microelectron. J., vol. 27, pp. 633-645, 1996 